\title{
Simulation of Pedestrian Crossing Behaviors at Unmarked Roadways Based on Social Force Model
}

\author{
Cao Ningbo, Wei Wei, Qu Zhaowei, Zhao Liying, and Bai Qiaowen \\ College of Transportation, Jilin University, Changchun 130022, China \\ Correspondence should be addressed to Wei Wei; weiwei@jlu.edu.cn
}

Received 25 May 2017; Revised 31 July 2017; Accepted 21 August 2017; Published 27 September 2017

Academic Editor: Paolo Renna

Copyright (c) 2017 Cao Ningbo et al. This is an open access article distributed under the Creative Commons Attribution License, which permits unrestricted use, distribution, and reproduction in any medium, provided the original work is properly cited.

\begin{abstract}
Limited pedestrian microcosmic simulation models focus on the interactions between pedestrians and vehicles at unmarked roadways. Pedestrians tend to head to the destinations directly through the shortest path. So, pedestrians have inclined trajectories pointing destinations. Few simulation models have been established to describe the mechanisms underlying the inclined trajectories when pedestrians cross unmarked roadways. To overcome these shortcomings, achieve solutions for optimal design features before implementation, and help to make the design more rational, the paper establishes a modified social force model for interactions between pedestrians and vehicles at unmarked roadways. To achieve this goal, stop/go decision-making model based on gap acceptance theory and conflict avoidance models were developed to make social force model more appropriate in simulating pedestrian crossing behaviors at unmarked roadways. The extended model enables the understanding and judgment ability of pedestrians about the traffic environment and guides pedestrians to take the best behavior to avoid conflict and keep themselves safe. The comparison results of observed pedestrians' trajectories and simulated pedestrians' trajectories at one unmarked roadway indicate that the proposed model can be used to simulate pedestrian crossing behaviors at unmarked roadways effectively. The proposed model can be used to explore pedestrians' trajectories variation at unmarked roadways and improve pedestrian safety facilities.
\end{abstract}

\section{Introduction}

Unlike pedestrians at the marked and signalized roadways, pedestrians at unmarked roadways are not restricted by the crosswalk boundary, so their trajectories have a wider variation, which results in a wider range distribution of conflict points. Pedestrians will take more complex stop/go decision-making and conflict avoidance mechanisms to cross the road quickly and safely. Pedestrians can wait at the lane line and the curb or bypass one vehicle from the front or behind the vehicle. Besides, pedestrians are attracted by traffic attraction point, such as bus station and market. Pedestrian prefer to choose the shortest path to the destination, which results in inclined crossing trajectory at unmarked roadways.

Many models have been put forward to model the crossing process of pedestrians, such as social force model, discrete choice model, cellular automata model, and gap-acceptancebased models. Microsimulation model can reproduce many complex traffic phenomena, and it is an effective method to assess new traffic design scheme. Cellular automata (CA) and social force model are two methods used frequently, and their effectiveness has been fully verified. Existing CA models related to pedestrians focus on the roadway conditions [1], traffic environment [2], or pedestrian movement mechanism [3]. In order to make the CA model more appropriate for pedestrians crossing a street, Feng et al. [4] put forward an improved cellular automata (CA) model. Considering the following characteristic of pedestrians when they cross a roadway, Zhou et al. [5] modeled the conformity tendency of pedestrians to make the CA model more realistic. CA model is not only used to simulate the behaviors of pedestrians, but also used to model the interactions between pedestrians and other traffic participants. Over the past years, many modified CA models have been established to simulate the interaction process between pedestrians and vehicles. Considering the following characteristic of cars, a car-following model was put forwarded by Jin et al. [6]. They took the influences of waiting pedestrians on the vehicles into consideration. In 
order to develop a simulation model for pedestrian-car mixed traffic flow, Xin et al. [7] developed a modified CA model. The proposed model is used to simulate the pedestrian-car mixed traffic flow at an unsignalized mid-block crosswalk. Although CA model is efficient in simulating mixed traffic flow, it has a disadvantage which is hard to ignore. Jasti and Higgs [8] indicated that the CA model is "discrete in nature," so it is not suitable to model the continuous interaction of the mixed flow; that is, it may not efficiently reflect an unexpected macroscopic behavior. Besides, the accuracy of the CA model is determined by the cellular space division, so the simulation will be more accurate when the cellular space is divided into more cells. But, because the cellular space cannot be divided infinitesimally, so the simulated speeds and locations of individuals have many similarities. So, a continuous model (social force model) is established to simulate the pedestrian flow. Agent can change the magnitude and direction of his/her speed according to the resultant force acted on the individual at any time, so the simulated results are more consistent to practical traffic flow.

Considering more realistic behaviors, existing forcebased models have been developed by modifying the SFM established by Helbing and Molnár [9]. Besides, other models, such as gap-acceptance-based models, can be combined in social force model. The SFM quantifies interactions between pedestrians and outside environment as repulsion and attraction forces and then updates pedestrians' positions in each simulation time to achieve pedestrian movements. Based on the social force and gap-acceptance model, the paper developed an improved pedestrian microsimulation model. Social force model can reproduce successfully many pedestrian collective phenomena such as lane formation, oscillations at bottle-necks, faster-is-slower effects, and clogging at exit doors. Pretto et al. [10] combined the rulebased models and force-based models to analyze and model the interactions between pedestrians and cars at crossing points. Schönauer et al. [11] used SFM to formulate pedestrian behaviors and analyze the interactions of pedestrians with cars. They used a single track approach to model vehicle dynamics and adopted the game theoretic approach to avoid pedestrians and vehicles conflicts in shared space conditions. Li et al. [12] took the required space and the critical gaps with turning vehicles into consideration, and they proposed a stop/go decision mechanism to avoid conflict. Anvari et al. [13] modeled pedestrian-car shared space via rulebased social force model. $\mathrm{Li}$ and $\mathrm{Hu}$ [14] indicated that the simulated fundamental speed-volume diagram of the mixing traffic based on the social force model is closer to the actuals than CA model, although many theoretical models have been proposed to explore the laws of mixed traffic flow. However, existing studies paid little attention to pedestrian crossing behaviors at unmarked roadways; what is more, the complex interactions of pedestrians and vehicles at unmarked roadways cannot be described. Currently, there is no simulation model which can be directly applied to pedestrian crossing behaviors at unmarked roadways, because pedestrians at unmarked roadways have more flexible stop/go decision-making and conflict avoidance mechanism without restrictions from crosswalk and signal.
Based on the SFM, a modified and extended social force model of pedestrian-car mixed traffic flow at unmarked roadways which is capable of reproducing the behaviors and trajectories of pedestrians at unmarked road is established and it can be implemented in traffic design scheme assessment. The essential mutual interferences of cars and pedestrians are analyzed. The paper effectively combines the stop/ go decision-making model and conflict avoidance mechanism of pedestrians into social force model. In this paper, SFMs of single pedestrian flow, single vehicle flow, and pedestrian-car mixed traffic flow are presented in Section 3. In order to verify the effectiveness of the proposed model, a case is analyzed in Section 4. The conclusions are presented in the end of the paper.

\section{Social Force Model}

The basic social force model contains four basic forces: driving force $\vec{f}_{\alpha}^{0}(t)$, force from obstacles $\vec{f}_{o \alpha}(t)$, force from pedestrians $\vec{f}_{\alpha \beta}(t)$, and random fluctuation force $\vec{\varepsilon}_{\alpha}(t)$. $\overrightarrow{\mathcal{E}}_{\alpha}(t)$ represents diverse random behaviors which cannot be predicted, and it obeys normal distribution and is perpendicular to the desired speed direction. The main equations of SFM are shown in

$$
\frac{d v_{\alpha}(t)}{d t}=\vec{f}_{\alpha}^{0}(t)+\sum_{\beta} \vec{f}_{\alpha \beta}(t)+\sum_{o} \vec{f}_{o \alpha}(t)+\vec{\varepsilon}_{\alpha}(t)
$$

$\vec{\varepsilon}_{\alpha}(t)$ is expressed as

$$
\vec{\varepsilon}_{\alpha}(t)=\left\langle\vec{e}_{\alpha}^{0}(t), \vec{f}_{\alpha}(t)\right\rangle X \vec{e}_{\alpha}^{\text {norm }}(t) .
$$

Here, $\vec{e}_{\alpha}^{0}(t)$ is unit vector of the desired speed direction; $\vec{e}_{\alpha}^{\text {norm }}(t)$ is vertical unit vector of the desired speed direction.

In (2), $X \sim N(0,1)$, and its probability density function is shown in

$$
f(x)=\frac{1}{\sqrt{2 \pi}} \exp \left(\frac{-x^{2}}{2}\right) .
$$

Driving force drives pedestrian $\alpha$ to adjust actual speed $\vec{v}_{\alpha}(t)$ to move in the desired direction $\vec{e}_{\alpha}^{0}(t)$ at a desired speed $v_{\alpha}^{0}(t)$ within a relaxation time.

$$
\begin{aligned}
& \vec{f}_{\alpha}^{0}(t)=\frac{v_{\alpha}^{0}(t) \vec{e}_{\alpha}^{0}(t)-\vec{v}_{\alpha}(t)}{\tau_{\alpha}} \\
& \vec{e}_{\alpha}^{0}(t)=\frac{\vec{P}_{\alpha}^{d}(t)-\vec{P}_{\alpha}^{c}(t)}{\left|\vec{P}_{\alpha}^{d}(t)-\vec{P}_{\alpha}^{c}(t)\right|},
\end{aligned}
$$

where $\vec{f}_{\alpha}^{0}(t)$ is driving force of pedestrian $\alpha$ at time $t ; v_{\alpha}^{0}(t)$ is the quantity of desired speed of pedestrian $\alpha$ at time $t ; \vec{v}_{\alpha}(t)$ is current speed vector at time $t ; \tau_{\alpha}$ is the relaxation time of 
pedestrian $\alpha ; \vec{e}_{\alpha}^{0}(t)$ is the unit vector of the desired speed direction; $\vec{P}_{\alpha}^{d}(t)$ is the desired destination of pedestrian $\alpha$, and $\vec{P}_{\alpha}^{c}(t)$ is the current position of the pedestrian $\alpha$.

The interaction force between pedestrian $\alpha$ and $\beta$ is calculated by (6). The sociopsychological force $\vec{f}_{\alpha \beta}^{\text {soc }}(t)$ is to keep privacy or personal space from nearby pedestrians, and it can be expressed as (7). The physical force $\vec{f}_{\alpha \beta}^{\mathrm{PH}}(t)$ contains physical pressure and sliding friction when one pedestrian contacts the others, and it is expressed as (9).

$$
\begin{aligned}
& \vec{f}_{\alpha \beta}(t)=\vec{f}_{\alpha \beta}^{\mathrm{soc}}(t)+\vec{f}_{\alpha \beta}^{\mathrm{PH}}(t) \\
& \vec{f}_{\alpha \beta}^{\text {soc }}(t)=A_{\alpha \beta} \exp \left[\frac{\left(r_{\alpha \beta}-d_{\alpha \beta}\right)}{B_{\alpha \beta}}\right] \vec{n}_{\alpha \beta} F_{\alpha \beta} \\
& \vec{n}_{\alpha \beta}=\left(n_{\alpha \beta}^{1}, n_{\alpha \beta}^{2}\right)=\frac{\left\{\vec{P}_{\alpha}(t)-\vec{P}_{\beta}(t)\right\}}{d_{\alpha \beta}} \\
& \vec{f}_{\alpha \beta}^{\mathrm{PH}}(t)=K g\left(r_{\alpha \beta}-d_{\alpha \beta}\right) \vec{n}_{\alpha \beta} \\
& +k g\left(r_{\alpha \beta}-d_{\alpha \beta}\right) \Delta v_{\alpha \beta}^{t} \vec{t}_{\alpha \beta} \\
& \vec{t}_{\alpha \beta}=\left(-n_{\alpha \beta}^{2}, n_{\alpha \beta}^{1}\right) \\
& \Delta v_{\alpha \beta}^{t}=\left(\vec{v}_{\alpha}-\vec{v}_{\beta}\right) * \vec{t}_{\alpha \beta} \\
& g(x)= \begin{cases}x, & x>0 \\
0, & \text { otherwise }\end{cases}
\end{aligned}
$$

where $\vec{f}_{\alpha \beta}(t)$ is the force between pedestrians $\alpha$ and $\beta$; $A_{\alpha \beta}$ and $B_{\alpha \beta}$ are the strength coefficients to be estimated; $\vec{P}_{\alpha}(t)$, $\vec{P}_{\beta}(t)$ are the center coordinates of pedestrians $\alpha$ and $\beta$ at time $t ; r_{\alpha \beta}$ is the sum of the radius of pedestrians $\alpha$ and $\beta ; d_{\alpha \beta}$ is the distance between the centers of pedestrians $\alpha$ and $\beta$; $\vec{n}_{\alpha \beta}$ is the vector pointing from pedestrians $\alpha$ to $\beta$. $F_{\alpha \beta}$ is the anisotropic factor; $\Delta v_{\alpha \beta}^{t}$ is the speed difference of pedestrian $\alpha$ and $\beta$ in tangent direction; $\vec{t}_{\alpha \beta}$ is vector in tangent direction; $K$ and $k$ are very large constants.

$F_{\alpha \beta}$ was introduced by Helbing and Molnár [9], and it can be calculated by (13). When $\lambda_{\alpha}$ takes different values from 0 to $1, F_{\alpha \beta}$ means that pedestrian has larger influences on front pedestrian than behind pedestrian.

$$
\begin{aligned}
F_{\alpha \beta} & =\lambda_{\alpha}+\left(1-\lambda_{\alpha}\right) \frac{1+\cos \left(\varphi_{\alpha \beta}\right)}{2} \\
\cos \left(\varphi_{\alpha \beta}\right) & =-\vec{n}_{\alpha \beta} \vec{e}_{\alpha}(t) \\
\vec{e}_{\alpha}(t) & =\frac{\vec{v}_{\alpha}(t)}{\left\|\vec{v}_{\alpha}(t)\right\|} .
\end{aligned}
$$

Interaction force between pedestrians and obstacles also contains sociopsychological force $\vec{f}_{o \alpha}^{\text {soc }}(t)$ and physical force $\vec{f}_{\text {od }}^{\mathrm{PH}}(t)$. The interaction force between pedestrian $\alpha$ and obstacle is calculated by (16).

$$
\begin{aligned}
\vec{f}_{o \alpha}(t)= & \vec{f}_{o \alpha}^{\mathrm{soc}}(t)+\vec{f}_{o \alpha}^{\mathrm{PH}}(t) \\
\vec{f}_{o \alpha}^{\mathrm{soc}}(t)= & A_{o \alpha} \exp \left[\frac{\left(r_{o \alpha}-d_{o \alpha}\right)}{B_{o \alpha}}\right] \vec{n}_{o \alpha} \\
\vec{f}_{o \alpha}^{\mathrm{PH}}(t)= & K g\left(r_{o \alpha}-d_{o \alpha}\right) \vec{n}_{o \alpha} \\
& -k g\left(r_{o \alpha}-d_{o \alpha}\right) \Delta v_{o \alpha}^{t} \vec{t}_{o \alpha} \\
\Delta v_{o \alpha}^{t}= & \vec{v}_{\alpha} * \vec{t}_{o \alpha} .
\end{aligned}
$$

Here, $\vec{n}_{o \alpha}$ is the vector to the center of an obstacle; $\vec{t}_{o \alpha}$ is tangent direction vector.

\section{Modeling Vehicles and Pedestrians}

\subsection{Modified Social Force Model for Pedestrians}

3.1.1. Model Development. In the initial social force model, Helbing indicated that all pedestrians, boundaries, and barriers in all directions have the same influences on the moving pedestrians. However, pedestrians will adjust their behaviors according to the surrounding traffic environment and the locations of pedestrians and obstacles. The pedestrians and obstacles in the sight of the moving pedestrians have greater influences on the moving pedestrians' behaviors. To explain this behavioral mechanism, they introduced anisotropic factor $F_{\alpha \beta} . F_{\alpha \beta}$ is expressed in (20). And, $F_{\alpha \beta}$ is expressed in Figure 1.

$$
\begin{aligned}
F_{\alpha \beta} & =\lambda_{\alpha}+\left(1-\lambda_{\alpha}\right) \frac{1+\cos \left(\varphi_{\alpha \beta}\right)}{2} \\
\cos \left(\varphi_{\alpha \beta}\right) & =-\vec{n}_{\alpha \beta} \vec{e}_{\alpha}(t) \\
\vec{e}_{\alpha}(t) & =\frac{\vec{v}_{\alpha}(t)}{\left\|\vec{v}_{\alpha}(t)\right\|},
\end{aligned}
$$

where $\lambda_{\alpha}$ is anisotropic coefficient, $0 \leq \lambda_{\alpha} \leq 1 ; \vec{e}_{\alpha}(t)$ is unit vector in velocity direction at time $t ; \varphi_{\alpha \beta}$ is velocity angle of pedestrians $\alpha$ and $\beta$.

$F_{\alpha \beta}$ means that pedestrians in the sight of moving pedestrians have greater influences on the moving pedestrians' behaviors. When $\lambda_{\alpha}$ equals 1 , pedestrians in all directions have the same influences on the moving pedestrians. When $\lambda_{\alpha}$ is smaller than 1 , pedestrians in front of moving pedestrians have greater effects than the behind pedestrians.

Besides, $\vec{\varepsilon}_{\alpha}(t)$ represents diverse random behaviors which cannot be predicted, and it obeys normal distribution and is perpendicular to the desired speed direction. In Helbing's initial social force model, he assumed that pedestrians 


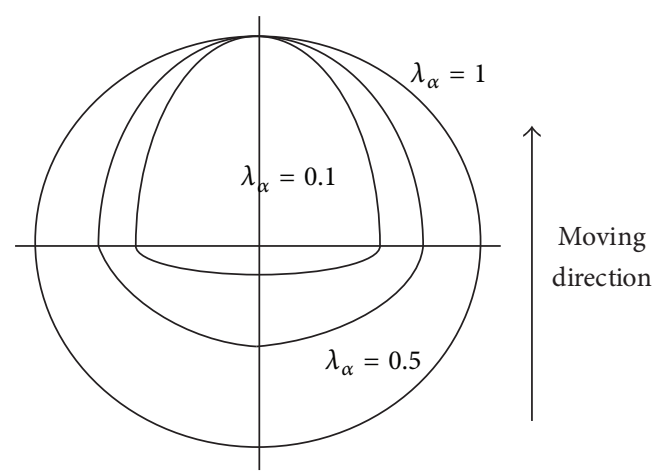

FIGURE 1: Schematic diagram of different anisotropic coefficients.

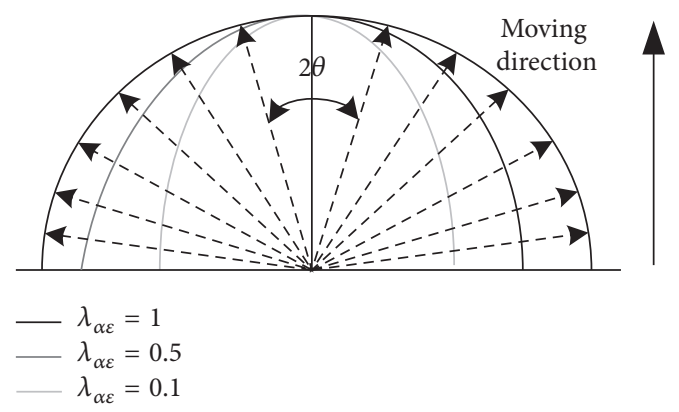

Figure 2: Possible speed directions and $F_{\alpha \varepsilon}$ after fluctuating.

may fluctuate in all directions. However, this assumption is not in conformity with the reality. Pedestrians will move forward without other disturbances and seldom move back, because they try to head to their destinations. So, a modified random fluctuation force $\vec{\varepsilon}_{\alpha}(t)$ is modeled. Similar to the anisotropic factor $F_{\alpha \beta}$, modified $\vec{\varepsilon}_{\alpha}(t)$ allows pedestrians to fluctuate within the field of vision. As shown in Figure 2, pedestrians fluctuate in the angle range of $2 \theta$, and $\theta$ changes from 0 to $\pi / 2$. The value of $\theta$ is calibrated by repeating pedestrian simulation. After pedestrians change their velocity directions to the proposed directions in Figure 2, their speeds will also change.

As expressed in (23), $F_{\alpha \varepsilon}$ is introduced to explain this phenomenon, and it represents the changes in direction and value of speed. After fluctuating, the velocity of pedestrian can be calculated by

$$
\begin{aligned}
F_{\alpha \varepsilon} & =\lambda_{\alpha \varepsilon}+\left(1-\lambda_{\alpha \varepsilon}\right) \frac{1+\cos \left(\varphi_{\alpha \varepsilon}\right)}{2} \\
\vec{v}_{\alpha \varepsilon}(t) & =\left\|\vec{v}_{\alpha}(t)\right\| \vec{e}_{\alpha \varepsilon}(t) F_{\alpha \varepsilon},
\end{aligned}
$$

where $\vec{v}_{\alpha \varepsilon}(t)$ is velocity of pedestrians after fluctuating; $\lambda_{\alpha \varepsilon}$ is anisotropic coefficient of diverse random behaviors, $0 \leq \lambda_{\alpha \varepsilon} \leq 1 ; \vec{e}_{\alpha \varepsilon}(t)$ is unit vector in velocity direction after fluctuating; $\varphi_{\alpha \varepsilon}$ is angle between velocity $\vec{v}_{\alpha}(t)$ before fluctuating and velocity $\vec{v}_{\alpha \varepsilon}(t)$ after fluctuating.

3.1.2. Stop/Go Decision-Making Model. Generally speaking, when vehicles arrive at the crossing area of pedestrians, pedestrians choose to cross the unmarked roadway without signal control by waiting the proper time gap in the vehicle traffic flow according to the complex traffic environment. However, when pedestrians have waited a long time, they tend to take adventure to cross the dense vehicle traffic flow. The interactions between pedestrians and vehicles become very severe. A stop/go decision-making model can enhance pedestrian capabilities of choosing proper crossing behaviors. Pedestrian crossing behaviors have been well described by some models, such as gap acceptance theory model. Brewer et al. [15] established a model to calculate the probability of pedestrian choosing proper time gap to cross. According to pedestrian crossing behavior model developed by Brewer et al., the probability of stop/go is related to headway gap of vehicles. In the Logit-based model, the error term of utility function to choose "go" is assumed to follow Gumbel distribution and the probability of going is calculated by

$$
p=\frac{e^{\mu \Delta T}}{1+e^{\mu \Delta T}} * 100 \longrightarrow \frac{1}{p} \propto e^{-\mu \Delta T},
$$

where $\Delta T$ is time gap between pedestrian and car.

$\mu$ equals 0.5 in this paper. $1 / p$ can be interpreted as a perceived risk $(\mathrm{PRv})$ from cars, pedestrian position on the road, and number of pedestrians. Known from (25), threats from cars are determined by time headway between two cars. When the time headway is large, threats from cars are small. For example, pedestrians do not want to cross the vehicle traffic flow when the time gap is small, and they choose to cross when the time gap is large enough.

Based on the gap acceptance theory, a stop/go decisionmaking model is established. At unsignalized and unmarked roadways, pedestrians change their crossing behaviors according to the time gap of cars, their locations, and group size. So, we try to develop a stop/go decision-making model by combining all these influence factors. The proposed stop/go decision-making model can describe the interactions between pedestrians and cars. Pedestrians will decide to go or not by weighing threats from vehicles, their locations, and group size. If the time needed by pedestrians to pass the conflict point is smaller than the critical gap time, they will cross. Otherwise, pedestrians will choose to wait until the proper time gap arises.

Known from (25), the probability of accepting a time gap is inversely proportional to perceived risk from cars. So, perceived risk from cars obeys the negative exponential form ( $\left.\mathrm{PRv} \propto e^{-\beta \Delta T}\right)$ indicated by Zhuang and $\mathrm{Wu}[16]$. The relationship between PRv and $e^{-\beta \Delta T}$ is shown in Figure 3. As shown in Figure 4, pedestrians are influenced by vehicles in different lanes, so we should calculate the total perceived risk from all cars in different lanes. However, vehicles in different lanes have different effects on the probability of accepting a time gap. So, a modified gap acceptance model considering which lane the car is located in should be established. The location and velocity of the headmost car in every lane can reflect the perceived risk from car. That is true for pedestrians standing still at the roadside. However, when crossing the roadway, pedestrians mainly focused on the time gap between cars in the nearest lane. For example, when crossing lane 


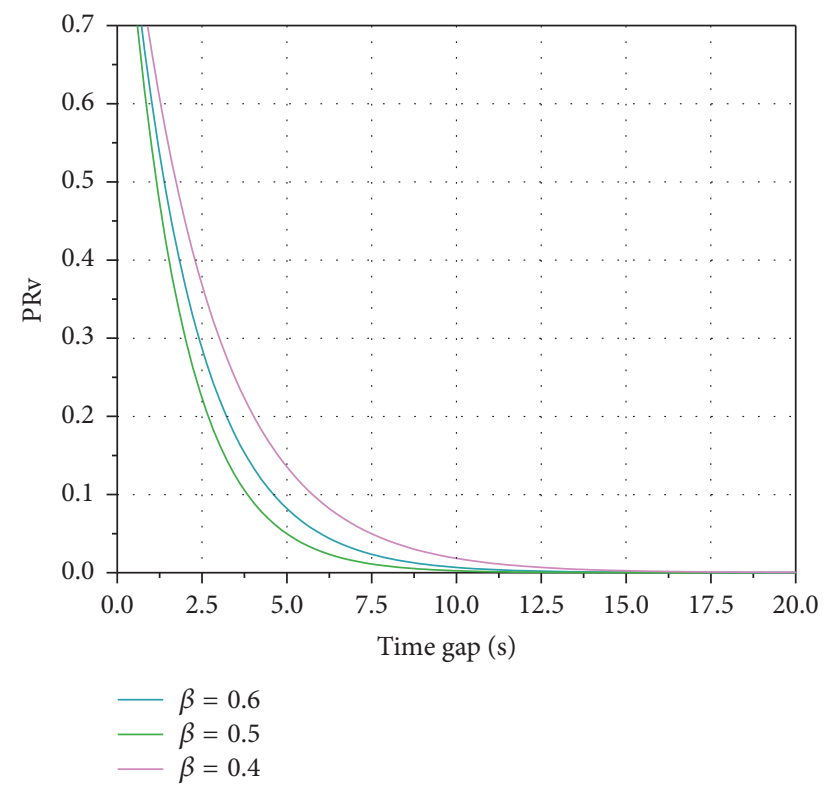

FIGURE 3: The relationship between perceived risk (PRv) and $\Delta T$.

1 , although the time gap is small in lane 3, pedestrians will still choose to cross the vehicle traffic flow in lane 1, because pedestrians assume that the cars in lane 3 have passed the conflict points when they arrive at lane 3. To make stop/go decision-making model more reasonable, threats from cars in different lanes should be taken into consideration. The first approaching car in every lane at the same half of the road with pedestrians is chosen to calculate the total perceived risk from cars. Among these approaching cars, the headmost vehicle in the nearest lane has the largest weight. After the headmost car has passed a pedestrian, the next car in the same lane becomes the new car to be chosen.

The total perceived risk PRv is expressed as (26),

$$
\begin{aligned}
\mathrm{PRv} & =\alpha_{\mathrm{PRv}} \sum_{i \in K} \frac{e^{-0.5|\Delta T|}}{y_{v i}-y_{p}+1} \\
K & =\left\{\frac{N}{2} * L \leq y_{\alpha} \leq y_{v i}+\frac{w}{2}, x_{v i} \geq x_{\alpha} \text { or } y_{\alpha}+\frac{w}{2}\right. \\
& \left.\leq \frac{N}{2} * L, x_{v i} \leq x_{\alpha}\right\}
\end{aligned}
$$

where $\alpha_{\mathrm{PRv}}$ helps to unite the scale; $K$ represents cars influencing pedestrians; $y_{v i}-y_{p}+1$ represents the adjustment for the perceived risk of cars in different lanes; $N$ is the number of lanes; $L$ is the lane width; $w$ is the vehicle width; $\left(x_{\alpha}, y_{\alpha}\right)$ is the coordinate of pedestrian $\alpha ;\left(x_{v i}, y_{v i}\right)$ is the coordinate of car $i$.

$\Delta T_{\alpha i}$ is the time difference between pedestrian $\alpha$ and car $i$ to the conflict point. It is expressed as

$$
\Delta T_{\alpha i}=\frac{\left\|\vec{P}_{c}(t)-\vec{P}_{i}(t)\right\|}{\left\|\vec{v}_{i}(t)\right\|} \cos \left\langle\vec{P}_{c}(t)-\vec{P}_{i}(t), \vec{v}_{i}(t)\right\rangle
$$

$$
\begin{aligned}
& -\frac{\left\|\vec{P}_{c}(t)-\vec{P}_{\alpha}(t)\right\|}{\left\|\vec{v}_{\alpha}(t)\right\|} \\
& \cdot \cos \left\langle\vec{P}_{c}(t)-\vec{P}_{\alpha}(t), \vec{v}_{\alpha}(t)\right\rangle,
\end{aligned}
$$

where $\vec{P}_{c}(t)$ is position vector of conflict point at time $t ; \vec{P}_{\alpha}(t)$ is position vector of pedestrian $\alpha$ at time $t ; \vec{P}_{i}(t)$ is position vector of car $i$ at time $t ; \vec{v}_{i}(t)$ is velocity of car $i$ at time $t ; \vec{v}_{\alpha}(t)$ is velocity of pedestrian $\alpha$ at time $t$.

Only $\Delta T_{i \alpha} \geq 0$ is effective, because $\Delta T_{\alpha i}<0$ means that car has passed the conflict point, and pedestrians cannot use this time gap.

Besides threats from cars, pedestrians also receive threats from their possible locations at next simulation time step (walk at the current speed). Perceived risk from locations (PRl) is determined by pedestrians' next positions on the road. Generally, road is divided into several lanes with white lines. Wang et al. [17] indicated that cars preferred to move in one lane at most time for safety. Pedestrians predict that the approaching cars move in the middle of the lane. Thus, it is assumed that pedestrians receive the highest perceived risk when their next positions locate at the middle line of the lane. Perceived risk decreases when pedestrians go away from the middle line of the lane. Usually, pedestrians run to the nearest lane boundary to decrease the perceived risk from approaching cars. A simple linear model is proposed to explain this phenomenon, and it is expressed as

$$
\mathrm{PRl}=\mathrm{PRl}_{\max }-a_{\mathrm{PRl}}\left|y_{p n}-\frac{y_{b 1}+y_{b 2}}{2}\right|,
$$

where $\alpha_{\mathrm{PRl}}$ helps to unite the scale; $\mathrm{PRl}_{\max }$ represents the maximum perceived risk form pedestrians locations, cars influencing pedestrians. $\left|y_{p n}-\left(y_{b 1}+y_{b 2}\right) / 2\right|$ represents the pedestrians' distance to the center of the current lane at the next simulation time step.

The probability of accepting a time gap is inversely proportional to perceived risk from cars and pedestrians' locations. It means that pedestrians will go when the perceived risk from cars and pedestrians' locations is low, and they will stop when the risk from cars and pedestrians' locations is high, because pedestrians prefer to choose the safest ways to cross the road. At the same time, pedestrians prefer to cross in group. Pedestrians think crossing will be more safe when the group size is large. Pedestrians who have the same moving destination, where the headway of the front pedestrian and the following pedestrian is not greater than $2 \mathrm{~s}$, are defined as a pedestrian group. Therefore, the total perceived risk from cars and pedestrians' locations should be adjusted by considering the group size. The modified total perceived risk model is expressed as

$$
\text { Perceived Risk }=\frac{\alpha_{g}}{N_{\text {group }}} *(\mathrm{PRv}+\mathrm{PRl}),
$$

where $\alpha_{g}$ helps to unite the scale; $N_{\text {group }}$ represents the pedestrian number of the group. 


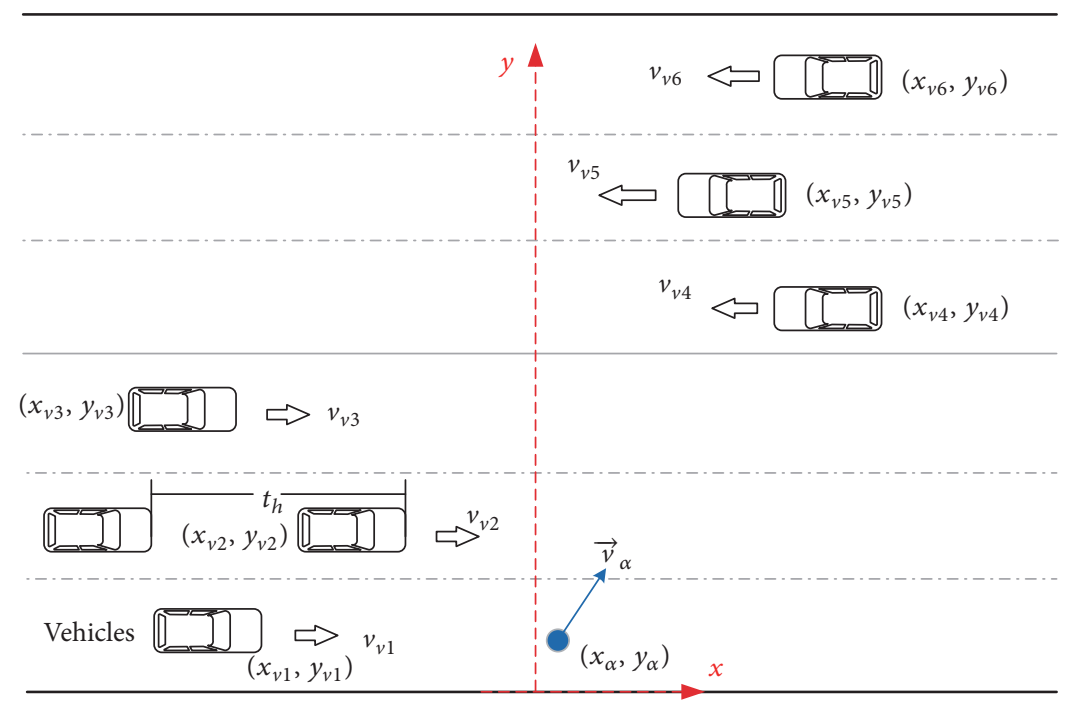

Figure 4: Schematic diagram of pedestrian crossing $(N=6)$.

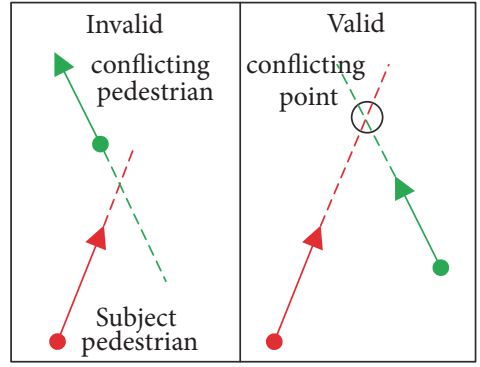

(a) Invalid and valid conflict

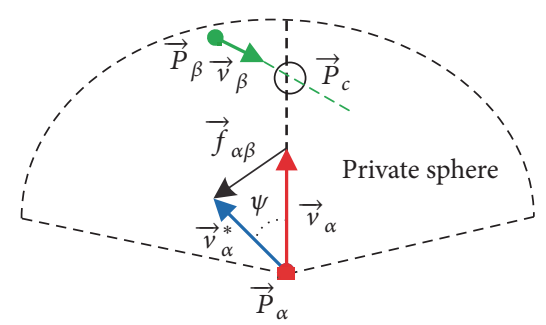

(b) Force from individual evasive behavior

Figure 5: Forces on individuals avoiding conflict.

In conclusion, the probability to go is expressed as

$$
P(\text { go })=\frac{1}{\text { Perceived Risk }}=\frac{N_{\text {group }}}{\alpha_{g} *(\mathrm{PRv}+\mathrm{PRl})} .
$$

3.1.3. Conflict Avoidance of Pedestrians. Asano et al. [18] assumed that the subject pedestrian would adopt a givingway maneuver to avoid collisions with the nearest pedestrian if they stepped into his/her private sphere, that is, if a collision was likely to occur. Two common conflicts are shown in Figure 5(a). However, since not all conflicts turn into collisions, we need to judge whether the conflicts are valid. As described in the study by Zeng et al. [19], the time to conflict point (TTCP) can be used to identify whether a conflict is valid or not. The TTCP represents the time it will take for two pedestrians to pass the conflict point at their current speeds and directions.

The TTCP for the subject pedestrian $\alpha$ and the conflicting pedestrian $\beta$ are calculated using (32). Only when the TTCPs of both pedestrians are positive, the conflict between them can be identified as valid. A negative TTCP value signifies that a pedestrian has passed the conflict point and no collision will occur.

$\operatorname{TTCP}_{\alpha}$

$$
=\frac{\left|\vec{P}_{c}(t)-\vec{P}_{\alpha}(t)\right|}{\left|\vec{v}_{\alpha}(t)\right|} \cos \left\langle\vec{P}_{c}(t)-\vec{P}_{\alpha}(t), \vec{v}_{\alpha}(t)\right\rangle
$$

$\operatorname{TTCP}_{\beta}$

$$
=\frac{\left|\vec{P}_{c}(t)-\vec{P}_{\beta}(t)\right|}{\left|\vec{v}_{\beta}(t)\right|} \cos \left\langle\vec{P}_{c}(t)-\vec{P}_{\beta}(t), \vec{v}_{\beta}(t)\right\rangle,
$$

where $\vec{P}_{\alpha}(t)$ is the position of pedestrian $\alpha, \vec{P}_{\beta}(t)$ is the position of pedestrian $\beta$, and $\vec{P}_{c}(t)$ is the position of the conflict point.

$T_{\alpha \beta}$ is the absolute value of the time difference of $\mathrm{TTCP}_{\alpha}$ and TTCP $_{\beta}$, and it is expressed as (33). $T_{\alpha \beta}$ can be used to 
evaluate the collision risk between two pedestrians. Larger values of $T_{\alpha \beta}$ signify lower collision risk.

$$
\begin{aligned}
& T_{\alpha \beta} \\
& = \begin{cases}\left|\mathrm{TTCP}_{\alpha}-\mathrm{TTCP}_{\beta}\right|, & \text { if } \mathrm{TTCP}_{\alpha}>0, \mathrm{TTCP}_{\beta}>0 \\
+\infty, & \text { otherwise. }\end{cases}
\end{aligned}
$$

Once a conflict is identified as valid, subject pedestrian $\alpha$ will adopt a giving-way maneuver to avoid collision with conflicting pedestrian $\beta$. As shown in Figure 5(b), the subject pedestrian avoids collision by adjusting his/her speed or direction. The giving-way maneuver guarantees that two pedestrians can go on moving after giving way rather than stopping forever due to force balance. In the real world, the giving-way maneuvers adopted by pedestrians are flexible and random. The pedestrians can pass by the conflict point by slowing down or speeding up. Pedestrian changing direction is a random behavior. Zeng et al. [19] proposed a stochastic adjustment mechanism to reflect direction changing. This paper also adopts this maneuver.

The adjusted speed vector $\vec{v}_{\alpha}^{*}(t)$ can be expressed by (34).

$$
\begin{aligned}
\vec{v}_{\alpha}^{*}(t) & =(1+\Delta v) \vec{v}_{\alpha}(t) \omega(\psi) \\
\omega(\psi) & =\left[\begin{array}{cc}
\cos \psi & -\sin \psi \\
\sin \psi & \cos \psi
\end{array}\right],
\end{aligned}
$$

where $\psi$ is the anticlockwise rotation angle; $\Delta v$ is the speed increment.

Therefore, the giving-way maneuver can be presented as an individual force $\vec{f}_{\alpha \beta}^{r}$ from the nearest conflicting pedestrian, and it can be calculated with

$$
\vec{f}_{\alpha \beta}^{r}=\frac{\vec{v}_{\alpha}^{*}-\vec{v}_{\alpha}}{\Delta t}
$$

\subsection{Social Force Model for Vehicles}

3.2.1. Social Force Model Extension of Vehicles. Helbing and Molnár [9] proposed that pedestrian can be represented by a circle with a radius $r_{\alpha}$, which represents the average space occupied by a pedestrian. They used an ellipse with the radius $r_{i}\left(\varphi_{i U}\right)$ to represent the average space occupied by a vehicle. As shown in Figure $6, r_{i}\left(\varphi_{i U}\right)$ is determined by the angle between the desired direction of vehicle $i$ and the speed direction of an approaching individual $(U=\alpha$ represents pedestrian and $U=j$ represents vehicle $). r_{i}\left(\varphi_{i U}\right)$ can be calculated with

$$
r_{i}\left(\varphi_{i U}\right)=\frac{w}{\sqrt{1-\varepsilon^{2} \cos \left(\varphi_{i U}\right)}}, \quad \text { where } \varepsilon=\frac{\sqrt{l^{2}-w^{2}}}{l}
$$

where $2 l$ represents the average vehicle length and $2 w$ represents the average vehicle width.

Similar to pedestrians, the basic social force model of vehicles contains driving force $\vec{f}_{i}^{0}(t)$, force from boundary
$\vec{f}_{B i}(t)$, force from other vehicles $\vec{f}_{i j}(t)$, and random fluctuation force $\vec{\varepsilon}_{i}(t)$. The modified SFM for cars in this paper also contains five basic social forces, $\vec{f}_{i}^{0}(t), \vec{f}_{B i}(t), \vec{f}_{i j}(t), \vec{\varepsilon}_{i}(t)$, and $\vec{f}_{\alpha i}(t)$ which is added to represent the force acted on the vehicles by pedestrians. The modified SFM is shown in

$$
\frac{d v_{i}(t)}{d t}=\vec{f}_{i}^{0}(t)+\vec{f}_{i j}(t)+\vec{f}_{B i}(t)+\vec{f}_{\alpha i}(t)+\vec{\varepsilon}_{i}(t) .
$$

The formula of $\vec{\varepsilon}_{i}(t)$ is expressed as

$$
\vec{\varepsilon}_{i}(t)=\left\langle\vec{e}_{i}(t), \vec{f}_{i}(t)\right\rangle X \vec{e}_{i}^{0}(t)
$$

The driving force of vehicle $i$ at time $t$ is expressed as (40), and it means that a force drives vehicle $i$ to adapt actual speed $\vec{v}_{i}(t)$ to move in the desired direction $\vec{e}_{i}^{0}(t)$ with a desired speed $v_{i}^{0}(t)$ within a relaxation time.

$$
\begin{aligned}
& \vec{f}_{i}^{0}(t)=\frac{v_{i}^{0}(t) \vec{e}_{i}^{0}(t)-\vec{v}_{i}(t)}{\tau_{i}} \\
& \vec{e}_{i}^{0}(t)=\frac{\vec{P}_{i}^{d}(t)-\vec{P}_{i}^{c}(t)}{\left|\vec{P}_{i}^{d}(t)-\vec{P}_{i}^{c}(t)\right|},
\end{aligned}
$$

where $\vec{f}_{i}^{0}(t)$ is driving force of vehicle $i$ at time $t ; v_{i}^{0}(t)$ is the quantity of desired speed of vehicle $i \vec{v}_{i}(t)$ is the speed vector at time $t ; \tau_{i}$ is the relaxation time of vehicle $i ; \vec{e}_{i}^{0}(t)$ is the unit vector of the desired speed direction; $\vec{P}_{i}^{d}(t)$ is the desired destination of vehicle $i$, and $\vec{P}_{i}^{c}(t)$ is the current position of vehicle $i$.

Vehicles are influenced by other vehicles within its visible range. The interaction force between vehicle $i$ and vehicle $j$ is calculated by (42). The sociopsychological force $\vec{f}_{i j}^{\text {soc }}(t)$ can be expressed as (43). $\vec{f}_{i j}^{\text {following }}(t)$ represents the force that appears when a vehicle takes measures to avoid conflict when the front vehicle slows down or speeds up.

$$
\begin{aligned}
& \vec{f}_{i j}(t)=f_{i j}^{\text {soc }}(t)+f_{i j}^{\text {following }}(t) \\
& f_{i j}^{\text {soc }}(t)=A_{i j} \exp \left[\frac{\left(r_{i j}-d_{i j}\right)}{B_{i j}}\right] \vec{n}_{i j} F_{i j},
\end{aligned}
$$

where $\vec{n}_{i j}$ represents the normalized vector pointing from car $i$ to car $j$; $A_{i j}$ represents force strength; $B_{i j}$ represents force distance; $d_{i j}$ represents the relative distance between the centers of cars; $r_{i j}$ represents the sum of their radius.

Vehicles are restricted in lanes, so lateral movement is impossible. The influencing range is explained in Figure 7. Similar to $F_{\alpha \beta}$, the anisotropic coefficient $F_{i j}$ is introduced to describe the phenomenon that the right ahead moving cars have greater influences on the moving cars than those cars in 


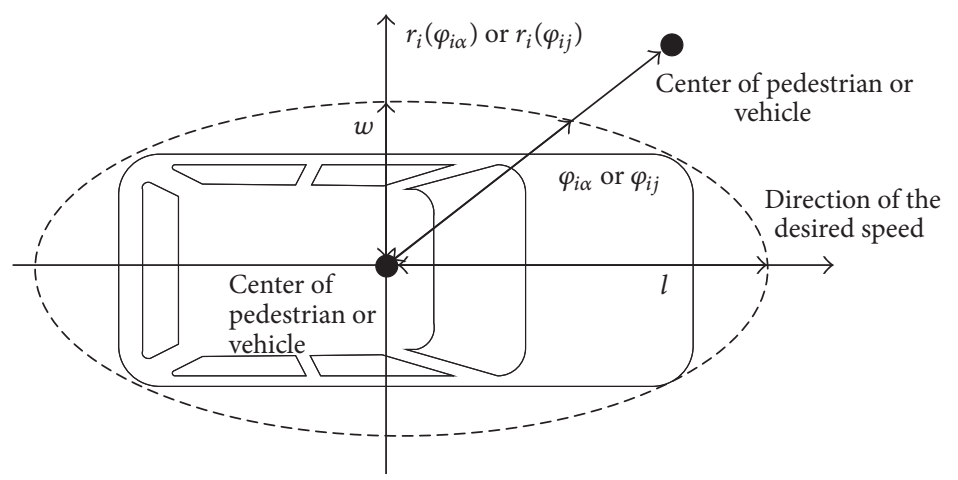

FIGURE 6: Schematic diagram of vehicle modeling.

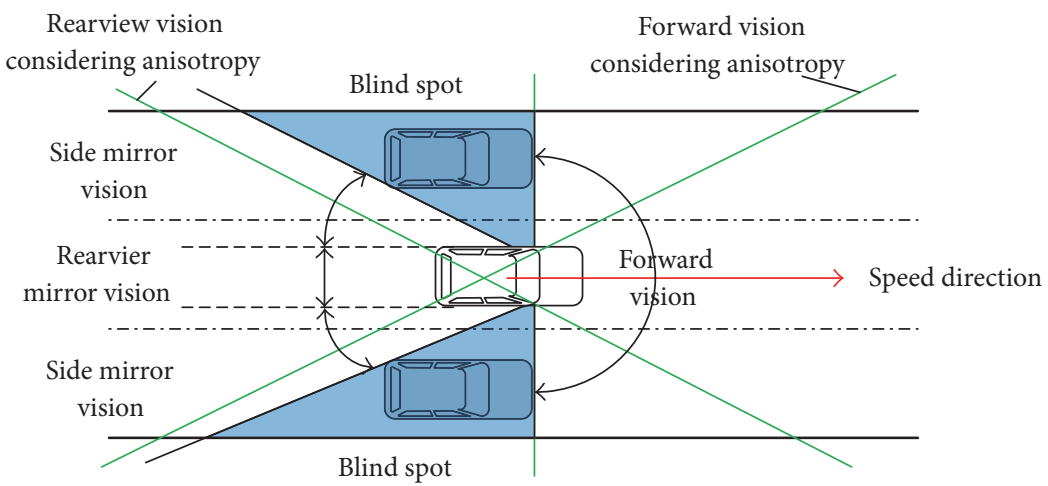

FIGURE 7: Influence range of vehicle considering vision.

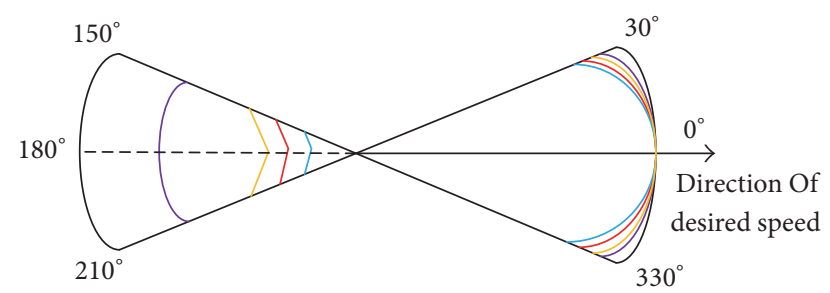

$\begin{array}{rlrl}-\lambda_{i j} & =0.05 & -\lambda_{i j}=1 \\ -\lambda_{i j} & =0.6 & -\lambda_{i j}=0.2 \\ -\lambda_{i j} & =0.13 & & \end{array}$

FIgURE 8: Schematic diagram of $F_{i j}$.

side front. $F_{i j}$ makes the social force model more realistic. $F_{i j}$ is explained in Figure 8, and it is expressed as (44).

$$
\begin{aligned}
& F_{i j}=\left(\lambda_{i j}+\left(1-\lambda_{i j}\right) \frac{1+\cos \left(\varphi_{i j}\right)}{2}\right) * p_{1} \\
& p_{1}= \begin{cases}1, & \text { if }-30^{\circ} \leq \varphi_{i j} \leq 30^{\circ}, 150^{\circ} \leq \varphi_{i j} \leq 210^{\circ} \\
0, & \text { otherwise. }\end{cases}
\end{aligned}
$$

And the moving vehicle is influenced by both the front vehicle and behind vehicle. So, $p_{1}$ is introduced to describe this phenomenon.
Besides, different from pedestrians, vehicles prefer to follow the front vehicle. Generally, the vehicles only move in the vehicle lane, and one vehicle lane allows one vehicle to cross at one moment. Compared with pedestrians, the vehicles are queue. So the following characteristic of vehicles is more remarkable, and it becomes more noticeable with the cars increasing. Anvari et al. (2012) indicated that the social force may cause the behind vehicle to overtake rather than follow the leading vehicle. If the following characteristic is not taken into consideration, the behind vehicle with a higher speed will attempt to overtake the leading vehicle instead of slowing down when the leading vehicle decelerates. Similarly, the behind vehicle with a lower speed will attempt to speed up instead of keeping its current speed when the leading vehicle accelerates.

To model the following behavior, Helbing et al. (1998) proposed a force $f_{i j}^{\text {following }}(t)$ in (46). In other words, the following car will take measures (adjust the speed) to react to the deceleration or acceleration behavior of the leading vehicle. Because vehicle only follows the leading vehicle, so $p_{2}$ is introduced to describe this phenomenon.

$$
\begin{gathered}
f_{i j}^{\text {following }}(t)=\left(-\frac{v_{i}^{0}(t) \vec{e}_{i}}{\tau_{i}} \exp \left(\frac{d\left(v_{i j}\right)-d_{i j}}{B_{i j}^{\prime}}\right)\right. \\
\left.-\frac{\Delta v_{i j}}{\tau_{i}^{\prime}} \exp \left(\frac{d\left(v_{i j}\right)-d_{r \delta}}{B_{i j}^{\prime \prime}}\right) \Theta\left(v_{i}\right)\right) * p_{2} * p_{3}
\end{gathered}
$$



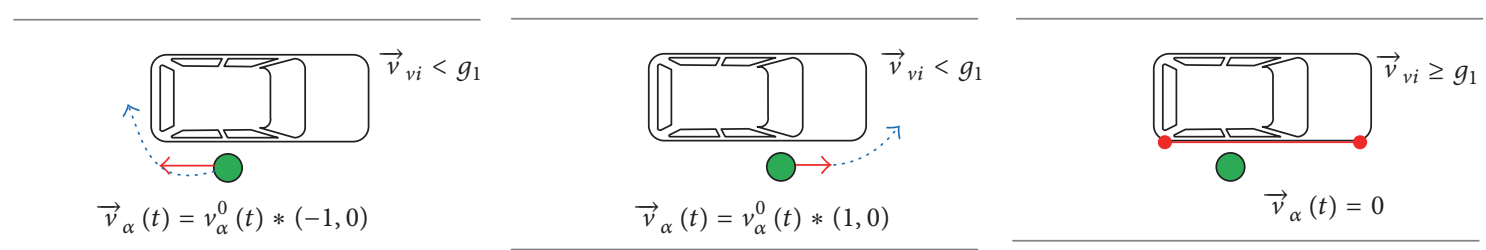

FIGURE 9: Conflict avoidance mechanism of pedestrians.

$$
\begin{aligned}
& \Theta\left(v_{i}\right)= \begin{cases}1, & \text { if } v_{i}>0 \\
0, & \text { otherwise }\end{cases} \\
& p_{2}= \begin{cases}1, & \text { if } 150^{\circ} \leq \varphi_{i j} \leq 210^{\circ} \\
0, & \text { otherwise. }\end{cases}
\end{aligned}
$$

However, the force $f_{i j}^{\text {following }}(t)$ does not exist all the time. When the distance between the behind car and the leading car is too far (larger than the constant $d_{1}$ ), the leading car has no influence on the behind car because the behind car has enough time to keep its current velocity. So, we add a coefficient $p_{3}$ to decide whether the force $f_{i j}^{\text {following }}(t)$ exists or not. $p_{3}$ can be expressed as

$$
p_{3}= \begin{cases}1, & \text { if } d_{i j}<d_{1} \\ 0, & \text { if } d_{i j} \geq d_{1} .\end{cases}
$$

The interaction force $\vec{f}_{\alpha i}(t)$ between pedestrian $\alpha$ and car $i$ is expressed as (50).

$$
\begin{aligned}
\vec{f}_{\alpha i}(t) & =A_{\alpha i} \exp \left[\frac{\left(r_{\alpha i}-d_{\alpha i}\right)}{B_{\alpha i}}\right] * \vec{n}_{\alpha i} * F_{\alpha i} \\
F_{\alpha i} & =\left(\lambda_{\alpha i}+\left(1-\lambda_{\alpha i}\right) \frac{1+\cos \left(\varphi_{\alpha i}\right)}{2}\right) * p_{4} \\
p_{4} & = \begin{cases}1, & \text { if }-30^{\circ} \leq \varphi_{\alpha i} \leq 30^{\circ} \\
0, & \text { otherwise, }\end{cases}
\end{aligned}
$$

where $\vec{n}_{\alpha i}$ represents the normalized vector pointing from car $i$ to pedestrian $\alpha ; A_{\alpha i}$ represents force strength; $B_{\alpha i}$ represents force distance; $d_{\alpha i}$ represents the relative distance between the centers of car and pedestrian; $r_{\alpha i}$ represents the sum of their radius.

The interaction force $\vec{f}_{B i}(t)$ between lane line $B$ and car $i$ is expressed as

$$
\vec{f}_{B i}(t)=A_{B i} \exp \left[\frac{r_{B i}}{B_{B i}}\right] * \vec{n}_{B i}
$$

where $\vec{n}_{B i}$ represents the normalized vector pointing from car $i$ to lane line $B ; A_{B i}$ represents force strength; $B_{B i}$ represents force distance; $r_{B i}$ represents the perpendicular distance from the center of car to the lane line.
3.2.2. Conflict Avoidance Model of Pedestrians and Vehicles. When a pedestrian moves into the road, if the vehicle or pedestrian does not decelerate immediately, a collision will happen. Once the conflict occurs, pedestrian $\alpha$ will adopt a giving-way maneuver to avoid collision with the conflicting car $i$. As shown in Figure 9, when there exists vehicle rather than time gap in front of pedestrians, pedestrians may bypass the vehicle from behind or front of vehicle. Note that pedestrians bypass the vehicle from the front of it only when vehicle moves at a low speed. When vehicle moves at a high speed, pedestrians will wait at the lane line to keep themselves safe.

So, an effective giving-way mechanism to describe such states is necessary. In the real world, the giving-way maneuvers adopted by pedestrians are flexible. The pedestrians can bypass the vehicle in front of them from the front or behind by changing moving direction. At the same time, changing moving direction depends on the relative location of pedestrian and car. This paper develops a conflict avoidance model to explain this maneuver of pedestrian. The conflict avoidance model of pedestrians and vehicles is expressed as

$$
\vec{v}_{\alpha}(t)= \begin{cases}0, & v_{v i} \geq g_{1} \\ {\left[v_{\alpha}^{0}(t), 0\right],} & v_{v i}<g_{1}, x_{\alpha}-x_{v i}>0 \\ {\left[-v_{\alpha}^{0}(t), 0\right],} & v_{v i}<g_{1}, x_{\alpha}-x_{v i} \leq 0\end{cases}
$$

where $g_{1}$ represents a critical constant.

\section{Calibration and Simulation}

4.1. Calibration of Model Parameters. As shown in Figure 10, one unmarked roadway near a bus station in Changchun City of China was selected as the study site. The width of the unmarked roadway is $27 \mathrm{~m}$. And the investigation is conducted from 13:00 to 15:00 in two consecutive working days. There are two bus stops around the studied site, as shown in Figure 10. The software based on video image processing system and developed by Jiang (2012) is applied to extract pedestrian and vehicle spatiotemporal data. The coordinates of pedestrians, speeds, and velocities were manually obtained from the video every $0.5 \mathrm{~s}$. The details of the data collection procedure and process are introduced by Cao et al. [20]. On the basis of pedestrian spatiotemporal data, pedestrian trajectories are acquired. In total, the crossing trajectories of 1023 pedestrians and 517 vehicles were obtained, and database contains 113,732 coordinates of pedestrians and cars. Velocities and acceleration can be obtained directly. Distances and velocity angles between pedestrians can be derived by calculation (distance formula of two points and 


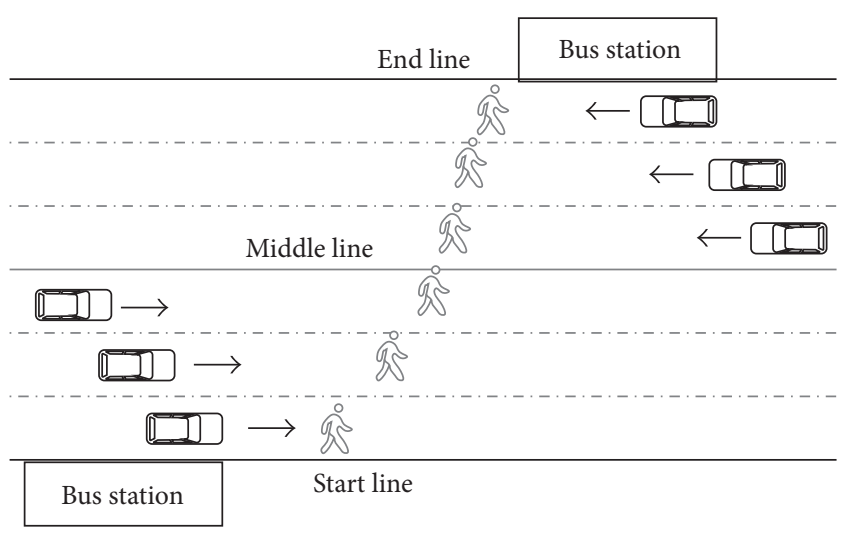

Figure 10: Schematic diagram of studied site.

angle formula of two vectors). There are sufficient velocities, acceleration, and coordinates data to enable a thorough analysis of pedestrians and vehicles motions.

The maximum log-likelihood estimation method was used by Zeng et al. [19] to calibrate the parameters of modified SFM. The calibration method used in this paper is based on the method adopted by Zeng et al. [19]. There are many parameters in the modified SFM. Some of the parameters can be obtained from the observed data directly, but others cannot.

In the study of Zeng et al. [19], a three-stage process is adopted to calibrate the parameters:

(1) The parameters $K, k, \lambda_{\alpha}$, and $r_{i}$, which are measurable but difficult to identify from the trajectory dataset, are estimated by referring to the research results of Anvari et al. [13], Zeng et al. [19], and Li et al. [21].

(2) Those parameters that can be determined from the observed dataset, for example, relaxation time of $\tau_{\alpha}$, $\tau_{i}, v_{\alpha}^{0}(t)$, and $v_{i}^{0}(t)$, are estimated directly. $v_{\alpha}^{0}(t)$ and $v_{i}^{0}(t)$ are the desired speed, and they take the average speeds.

(3) After determining these measurable parameters, nonmeasurable parameters, such as the strength coefficients that do not have concrete physical meanings, are derived by the maximum log-likelihood estimation method.

Real trajectories are extracted from video data of studied unmarked road. The trajectory extractor software developed by Jiang Sheng was used to extract the trajectories, velocities, and acceleration of pedestrians from video. The video analysis procedure was explained by Jiang (2012), and the error and accuracy of the software were analyzed in detail. Other nonmeasurable parameters, such as $A_{\alpha \beta}, B_{\alpha \beta}, A_{o \alpha}, B_{o \alpha}, F$, $\eta$, and $g_{i}$, are calibrated by the maximum log-likelihood estimation method. The position of pedestrian $\alpha$ at next time step $\left(\vec{P}_{\alpha}\left(t_{k+1} \mid \theta_{p}\right)\right)$ is determined by the resultant force, and the resultant force closely depends on the SFM parameters $\left(\theta_{p}\right.$, which represents the set of all model parameters, $A_{\alpha \beta}$, $B_{\alpha \beta}, A_{o \alpha}, B_{o \alpha}$, and so on). The distance vector $\Delta \vec{d}_{\alpha}^{\text {est }}\left(t_{k} \mid \theta_{p}\right)$ points from $\vec{P}_{\alpha}\left(t_{k} \mid \theta_{p}\right)$ to $\vec{P}_{\alpha}\left(t_{k+1} \mid \theta_{p}\right)$. Here, the $x$ and $y$ components of $\Delta \vec{d}_{\alpha}^{\text {est }}\left(t_{k} \mid \theta_{p}\right)$ are assumed to obey a normal distribution with mean $\left(\mu_{x}, \mu_{y}\right)$ and standard deviation $\left(\sigma_{x}, \sigma_{y}\right) .\left(\mu_{x}, \mu_{y}\right)$ and $\left(\sigma_{x}, \sigma_{y}\right)$ can be calculated from the observed $\Delta \vec{d}_{\alpha}^{\text {obs }}\left(t_{k} \mid \theta_{p}\right)$. In a single prediction step (from $t_{k}$ to $t_{k+1}$ ), the likelihood $L_{k}$ is expressed as

$$
L_{k}\left(\Delta d_{\alpha}\left(\theta_{p}\right) \mid \mu, \sigma\right)=\frac{1}{\sigma \sqrt{2 \pi}} e^{-\left(\Delta d_{\alpha}\left(\theta_{p}\right)-\mu\right)^{2} / 2 \sigma^{2}} .
$$

Because $x$ and $y$ direction components are assumed to obey normal distributions, $\Delta d_{\alpha}\left(\theta_{p}\right)$ can be expressed as

$$
\Delta d_{\alpha}\left(\theta_{p}\right)=\left\|\Delta \vec{d}_{\alpha x}^{\mathrm{est}}\left(t_{k} \mid \theta_{p}\right)\right\|+\left\|\Delta \vec{d}_{\alpha y}^{\mathrm{est}}\left(t_{k} \mid \theta_{p}\right)\right\| .
$$

$\mu$ and $\sigma$ can be calculated using

$$
\mu=\mu_{x}+\mu_{y}
$$

$\sigma$

$$
=\sqrt{\sigma_{x}^{2}+\sigma_{y}^{2}+2 \operatorname{cov}\left(\left\|\Delta \vec{d}_{\alpha x}^{\text {est }}\left(t_{k} \mid \theta_{p}\right)\right\|,\left\|\Delta \vec{d}_{\alpha y}^{\text {est }}\left(t_{k} \mid \theta_{p}\right)\right\|\right)},
$$

where $\Delta \vec{d}_{\alpha x}^{\text {est }}\left(t_{k} \mid \theta_{p}\right)$ is the $x$ component of $\Delta \vec{d}_{\alpha}^{\text {est }}\left(t_{k} \mid \theta_{p}\right)$ and $\Delta \vec{d}_{\alpha y}^{\text {est }}\left(t_{k} \mid \theta_{p}\right)$ is the $y$ component of $\Delta \vec{d}_{\alpha}^{\text {est }}\left(t_{k} \mid \theta_{p}\right)$.

The $L_{k}\left(\Delta d_{\alpha}\left(\theta_{p}\right) \mid \mu, \sigma\right)$ of model parameter $\theta_{p}$ can be expressed as

$$
\begin{aligned}
L\left(\Delta d_{\alpha}\left(\theta_{p}\right) \mid \mu, \sigma\right) & =\prod \frac{1}{\sigma \sqrt{2 \pi}} e^{-\left(\Delta d_{\alpha}\left(\theta_{p}\right)-\mu\right)^{2} / 2 \sigma^{2}} \\
& =\frac{(2 \pi)^{-n / 2}}{\sigma^{n}} e^{-\sum\left(\Delta d_{\alpha}\left(\theta_{p}\right)-\mu\right)^{2} / 2 \sigma^{2}} .
\end{aligned}
$$

To simplify the process of computation, logarithms are taken of both sides of (58) in order to turn (58) to (59). The model parameter $\theta_{p}$ can be obtained by maximizing

$$
\begin{aligned}
\ln \left(L\left(\Delta d_{\alpha}\left(\theta_{p}\right) \mid \mu, \sigma\right)\right) \\
=-\frac{n}{2} \ln (2 \pi)-n \ln (\sigma)-\frac{\sum\left(\Delta d_{\alpha}\left(\theta_{p}\right)-\mu\right)^{2}}{2 \sigma^{2}} .
\end{aligned}
$$

The calibration results of parameters are presented in Table 1 . These parameters are applied to simulate the modified SFM. The $p$ values at the $95 \%$ confidence level indicate that all the parameters of the model are effective.

4.2. Example Analysis. A simulation analysis of pedestrians crossing unmarked road on Yatai Street in Changchun of China is made. The width of the road segment is $27 \mathrm{~m}$. In order to obtain stability simulation results, there are 573 vehicles and 713 pedestrians crossing the road segment. The simulation is repeated 100 times to eliminate the influence of random disturbance. The anisotropic characters of pedestrians and vehicles are set to 0.3 so that interactions outside of the field of view have little effect on pedestrians and vehicles. 

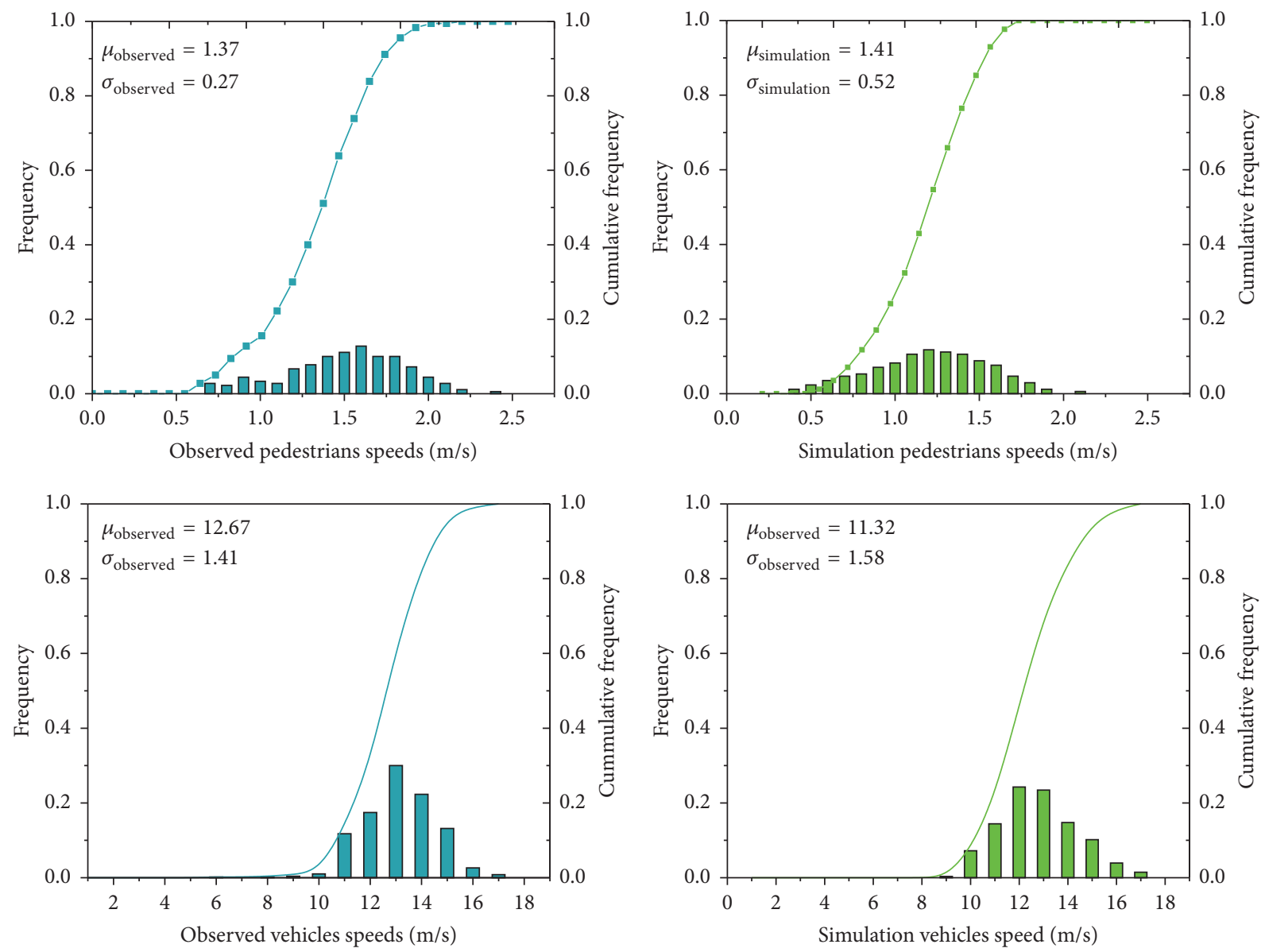

FIGURE 11: Speed histograms of pedestrians observed and simulation results.

After 100 simulations being done, all the average speeds of individuals (pedestrians or cars) in the simulation scenarios are recorded. Then, the simulation speed distribution and the observed speed distribution are compared. Figure 11 shows the analysis of the speed distributions of pedestrians and vehicles. It is clear that the simulation speed distribution and observed speed distribution match closely by comparing their mean $\mu$ and standard deviation $\sigma$, which indicates that the modified social force model is effective.

4.3. Comparison of Simulated and Observed Trajectories. Figure 12 shows the comparison of observed and simulated trajectories from 51 pedestrians in one crossing. After using two sample $t$-tests for the difference of crossing positions at three cross sections at a $95 \%$ confidence level, there is no significant difference according to the analysis result. The modified social force model successfully reproduced the pedestrian trajectories. The shape and outline of trajectories are quite similar, and the shortest paths to the bus station have attracted more pedestrians. This indicates that pedestrians prefer to head to the destination by the shortest paths.

But, it is not convincing enough if we only use the similar shape and outline of trajectories to show the effectiveness of the proposed model. We should validate the proposed model from more microscopic results. Generally speaking, comparing the observed and simulated trajectory of one pedestrian every time is the best method to prove the validity of the developed model. However, it needs considerable word, and it is unreasonable. Because, pedestrian behaviors are random and unpredictable, and we cannot determine which simulated trajectory should be selected to be compared with observed trajectory of one pedestrian. We only are able to validate the proposed model by comparing the available and observed characteristics of pedestrians. So, we select the distributions of crossing positions at two sections (middle line and end line) to validate the proposed model. By comparing the probability and cumulative probability of observed and estimated crossing positions at two sections, it is obvious that the distributions of estimated crossing positions agree with observed ones well. As shown in Figure 13, the average of the crossing positions at two cross sections is also very close. It means that estimated pedestrian trajectories variation agrees with the observed pedestrian trajectories variation well.

The above comparison (Figure 12) of observed and simulated trajectories only indicates that the proposed model is effective at macrolevel. A more detailed comparison of pedestrian observed and simulated typical trajectories is also made. The typical trajectories of pedestrians can be divided 
TABLE 1: Parameter calibration results.

\begin{tabular}{|c|c|c|c|}
\hline Parameters & Eq. & Estimates & $p$ value \\
\hline Strength coefficients for force from other pedestrians $A_{\alpha \beta}$ & $(7)$ & 0.75 & 0.03 \\
\hline Reaction distance for force from other pedestrians $B_{\alpha \beta}$ & $(7)$ & 1.75 & 0.04 \\
\hline Strength coefficients for force from other vehicles $A_{i j}$ & $(42)$ & 7 & 0.02 \\
\hline Reaction distance for force from other vehicles $B_{i j}$ & $(42)$ & 10 & 0.02 \\
\hline Strength coefficients for force from obstacle $A_{o \alpha}$ & (17) & 0.5 & 0.01 \\
\hline Reaction distance for force from obstacle $B_{o \alpha}$ & (17) & 4.7 & 0.04 \\
\hline Strength coefficients for force from boundary $A_{B i}$ & (53) & 2.7 & 0.05 \\
\hline Reaction distance for force from boundary $B_{B i}$ & (53) & 4.9 & 0.03 \\
\hline Strength coefficients for force between vehicle and pedestrian $A_{\alpha i}$ & $(50)$ & 5.3 & 0.03 \\
\hline Reaction distance for force between vehicle and pedestrian $B_{\alpha i}$ & $(50)$ & 5.7 & 0.07 \\
\hline Reaction distance for acceleration interaction $B_{i j}^{\prime}$ & $(46)$ & 5.59 & \\
\hline Reaction distance for braking interaction $B_{i j}^{\prime \prime}$ & $(46)$ & 8.62 & \\
\hline Constant $\alpha_{\mathrm{PR} 1}$ & $(29)$ & 1.7 & 0.03 \\
\hline Constant $\alpha_{g}$ & $(30)$ & 4.5 & 0.07 \\
\hline Constant $P R l_{\max }$ & $(29)$ & 3.4 & 0.05 \\
\hline Constant $d_{1} / \mathrm{m}$ & $(49)$ & 7.2 & 0.01 \\
\hline Constant $g_{1} / \mathrm{m} / \mathrm{s}$ & $(54)$ & 3.67 & 0.07 \\
\hline Radius of pedestrian $r_{\alpha}$ & / & 0.5 & \\
\hline Relaxation time of pedestrian $\tau_{\alpha} / \mathrm{s}$ & $(4)$ & 0.3 & \\
\hline Relaxation time of vehicle $\tau_{i} / \mathrm{s}$ & $(46)$ & 2.4 & \\
\hline Braking time of vehicle $\tau_{i}^{\prime}$ & $(46)$ & 0.77 & \\
\hline Simulation time step $\Delta t$ & $(36)$ & 0.06 & \\
\hline Anisotropic factor coefficient $\lambda_{\alpha}, \lambda_{\alpha \varepsilon}, \lambda_{i j}$ and $\lambda_{\alpha \varepsilon}$ & (13), (20), (23), (44), (51) & 0.3 & \\
\hline Desired speed of pedestrian $v_{\alpha}^{0} / \mathrm{m} / \mathrm{s}$ & $(4)$ & 1.37 & \\
\hline Desired speed of vehicle $v_{i}^{0} / \mathrm{m} / \mathrm{s}$ & $(30)$ & 14.7 & \\
\hline
\end{tabular}
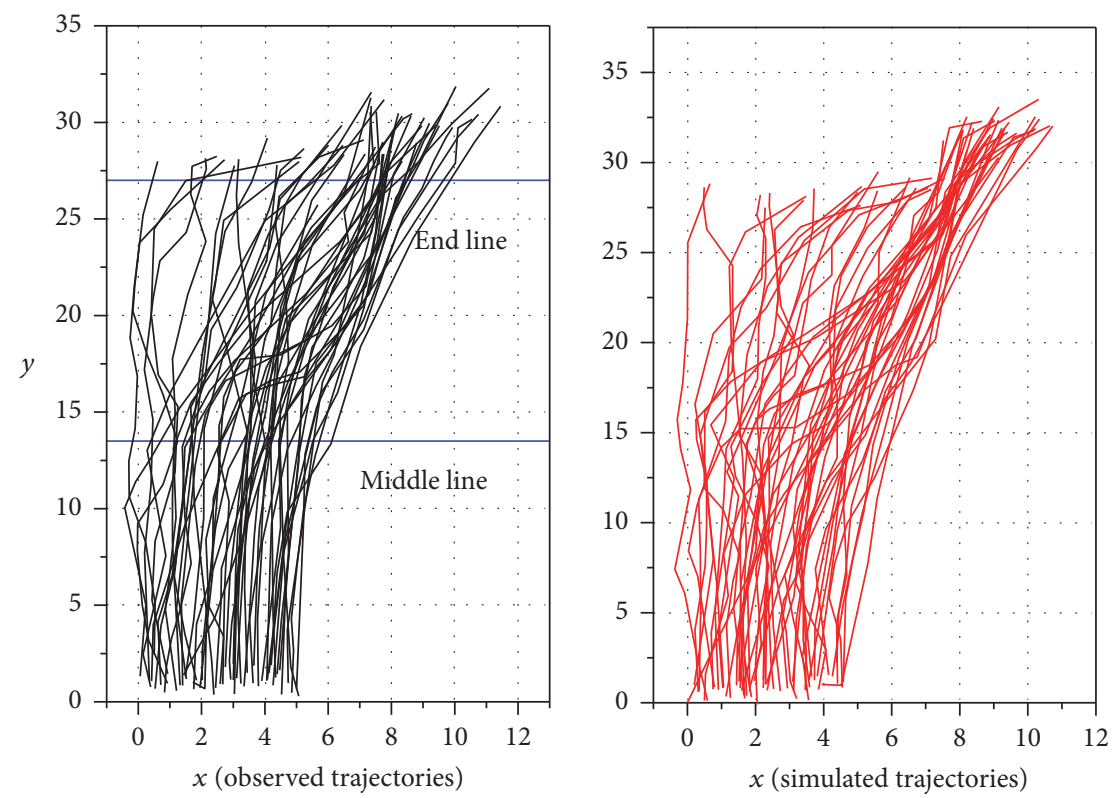

FIGURE 12: Comparison of simulated and observed trajectories. 

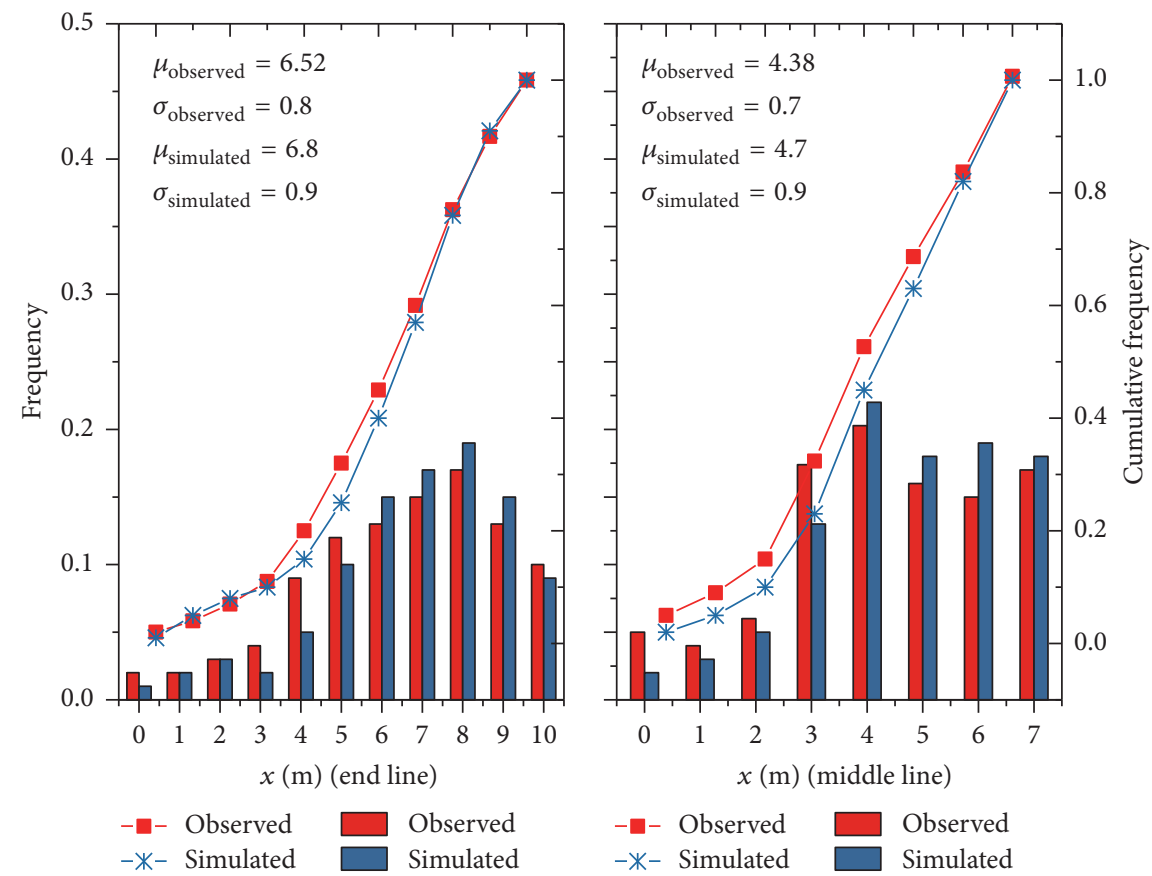

FIGURE 13: Comparison of simulated and observed crossing positions.

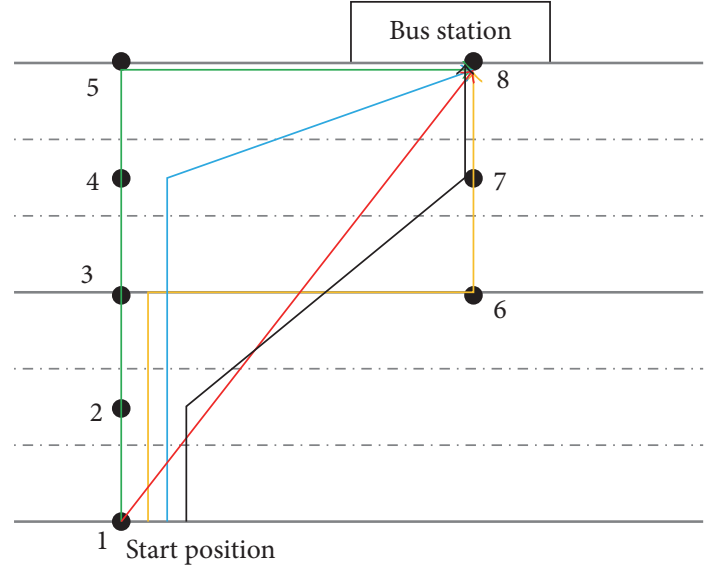

Path type

$\begin{array}{ll}\rightarrow \text { (a) } 1-2-3-4-5-8 & \rightarrow \text { (d) } 1-2-3-4-8 \\ \rightarrow \text { (b) } 1-3-6-8 & \rightarrow \text { (e) } 1-2-7-8 \\ \rightarrow \text { (c) } 1-8 & \end{array}$

Figure 14: Schematic diagram of five path types.

roughly into several categorizations. As shown in Figure 14, five typical path types are described. These paths are obtained by connecting a series of intermediate destinations. Point 1 is the start point, and Point 8 is the final destination. Other points represent intermediate destinations.

As shown in Figure 15, we compared five representative observed and simulated trajectory samples considering the start and end points. Then, we estimated the fitness of observed and simulated paths. The representative trajectories were chosen as examples to compare the observed and simulated trajectories because their fitness is closest to the average fitness of the path type. The root-mean-square errors (RMSE) of the lateral position of all paths are shown in Figure 15. It is clear that RMSEs increases with the pedestrians' end points becoming farther. The RMSE of type (a) is the smallest. Generally speaking, simulated trajectories are close to the observed trajectories. The fitting $R^{2}$ of observed and simulated trajectories was also calculated. Among all the types, the fitting $R^{2}$ of type (a) is the maximum and RMSE is the smallest, because pedestrians have limited variations in the lateral direction. Type (e) has the smallest $R^{2}$, and it means that type (e) was not reproduced well. That is because pedestrians may move in lateral direction to the destination, and pedestrians wait in the middle of the road instead of moving in lateral direction to the destination in simulation. So the simulated trajectories always go directly to the final destination instead of turning to the temporary destination first (see Figure 14). In summary, pedestrian trajectories were reproduced with small root-mean-square errors and acceptable $R^{2}$.

\section{Conclusions}

Based on social force model, a modified social force model considering the interactions between pedestrians and vehicles, conflict avoidance with cars, and group evasive was established. Then, the relevant parameters of the proposed social force model were calibrated by using maximum likelihood estimation method. Finally, the effectiveness of proposed models was verified by comparing the observed and simulated pedestrian trajectories at unmarked road. 


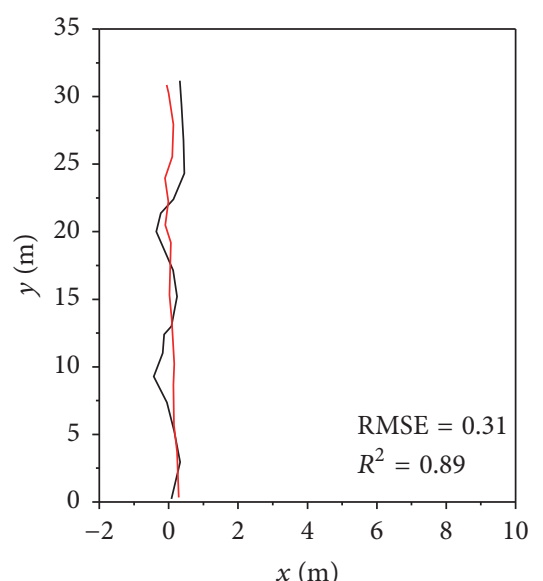

$x(\mathrm{~m})$

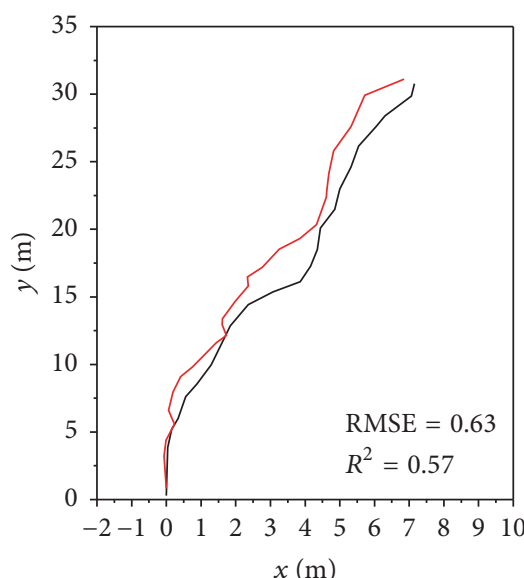

- Observed trajectory _ Simulated trajectory

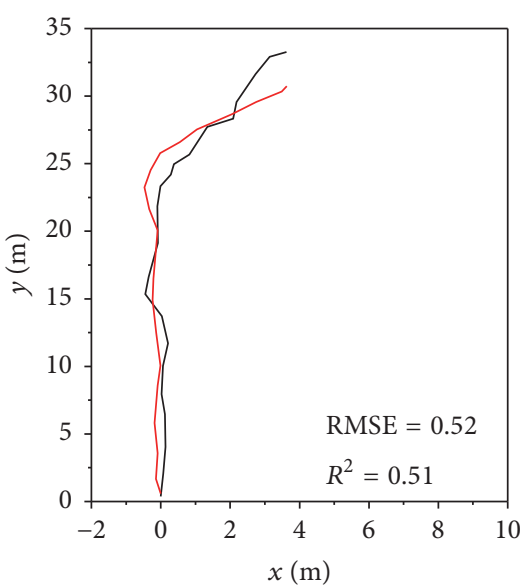

- Observed trajectory

Simulated trajectory

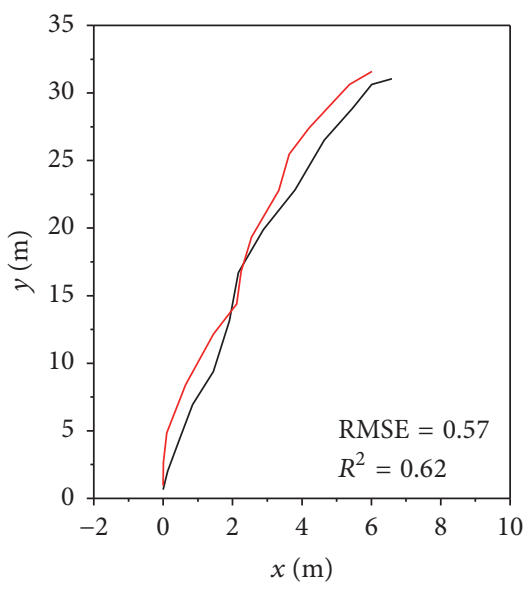

— Observed trajectory

— Simulated trajectory

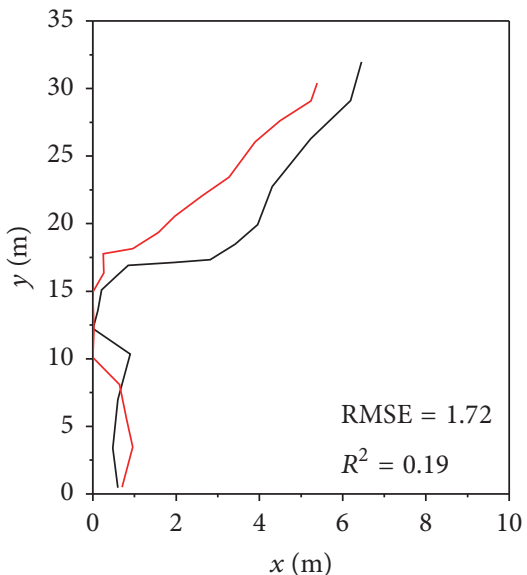

- Observed trajectory

— Simulated trajectory

FiguRE 15: Examples of simulated and observed pedestrian trajectories for five path types.

The major contributions and innovations of the paper are summarized as follows.

(1) The stop/go decision-making model was developed to describe the phenomenon that pedestrians cross the vehicle traffic flow.

(2) So far, most of the studies focused on modifying the social force model for pedestrians. And the mechanisms of interactions between pedestrians and vehicles were seldom taken into consideration, let alone modeled with social force model.

(3) A conflict avoidance model of pedestrians and vehicles was modeled. The bypassing maneuver adopted by pedestrians was modeled. The model enables pedestrians to cross the vehicle traffic flow effectively when vehicles move at low speeds.

The similar shape and outline of observed and simulated pedestrian trajectories, small root-mean-square errors, and acceptable $R^{2}$ illustrate the effectiveness of the modified social force model from different angles. However, several problems were not taken into consideration. For instance, the conflict avoidance model of pedestrians and vehicles is too simple, and pedestrians prefer to bypass from behind the vehicle when they cross the road in crowd, so a more reasonable conflict avoidance model should be established. These limitations will be overcome by developing a more reasonable model in our future work.

\section{Conflicts of Interest}

There are no conflicts of interest regarding the publication of this paper.

\section{Acknowledgments}

This research was funded by the National Natural Science Foundation of China (nos. 51278520 and 51278220). 


\section{References}

[1] M.-A. Granié, T. Brenac, M.-C. Montel, M. Millot, and C. Coquelet, "Influence of built environment on pedestrian's crossing decision," Accident Analysis and Prevention, vol. 67, pp. 7585, 2014.

[2] J. Yang, W. Deng, J. Wang, Q. Li, and Z. Wang, "Modeling pedestrians' road crossing behavior in traffic system microsimulation in China," Transportation Research Part A: Policy and Practice, vol. 40, no. 3, pp. 280-290, 2006.

[3] R. T. Bedeley, N. O. Attoh-Okine, and E. R. Lee, "Modelling pedestrian crossing behaviour using bayesian networks," Proceedings of the Institution of Civil Engineers: Transport, vol. 166, no. 5, pp. 282-288, 2013.

[4] S. Feng, N. Ding, T. Chen, and H. Zhang, "Simulation of pedestrian flow based on cellular automata: a case of pedestrian crossing street at section in China," Physica A: Statistical Mechanics and Its Applications, vol. 392, no. 13, pp. 2847-2859, 2013.

[5] R. Zhou, W. J. Horrey, and R. Yu, "The effect of conformity tendency on pedestrians' road-crossing intentions in China: an application of the theory of planned behavior," Accident Analysis \& Prevention, vol. 41, no. 3, pp. 491-497, 2009.

[6] S. Jin, X. Qu, C. Xu, and D. Wang, "Dynamic characteristics of traffic flow with consideration of pedestrians' road-crossing behavior," Physica A: Statistical Mechanics and Its Applications, vol. 392, no. 18, pp. 3881-3890, 2013.

[7] X. Xin, N. Jia, L. Zheng, and S. Ma, "Power-law in pedestrian crossing flow under the interference of vehicles at an unsignalized midblock crosswalk," Physica A: Statistical Mechanics and Its Applications, vol. 406, pp. 287-297, 2014.

[8] V. K. Jasti and C. F. Higgs, "A lattice-based cellular automata modeling approach for granular flow lubrication," Journal of Tribology, vol. 128, no. 2, pp. 358-364, 2006.

[9] D. Helbing and P. Molnár, "Social force model for pedestrian dynamics," Physical Review E Statistical Physics Plasmas Fluids and Related Interdisciplinary Topics, vol. 51, no. 5, article 4282, 1995.

[10] C. O. Pretto, H. B. B. Cybis, and A. Jacobsen, A multi-layer simulation model for vehicle and pedestrian interaction (No. 113833), 2011.

[11] R. Schönauer, M. Stubenschrott, W. Huang, C. Rudloff, and M. Fellendorf, "Modeling concepts for mixed traffic: Steps toward a microscopic simulation tool for shared space zones," Transportation Research Record: Journal of the Transportation Research Board, no. 2316, pp. 114-121, 2012.

[12] S. S. Li, D. L. Qian, and Y. Luo, "Microscopic dynamic simulation model for pedestrian at signalized intersection," Journal of Central South University, vol. 19, no. 11, pp. 3351-3362, 2012.

[13] B. Anvari, M. G. H. Bell, A. Sivakumar, and W. Y. Ochieng, "Modelling shared space users via rule-based social force model," Transportation Research Part C: Emerging Technologies, vol. 51, pp. 83-103, 2015.

[14] M.-S. Li and Y. Hu, "Analysis and comparison between cellular automata model and social force model of mixed traffic flow," Logistics Engineering and Management, no. 3, pp. 161-164, 2015.

[15] M. Brewer, K. Fitzpatrick, J. Whitacre, and D. Lord, "Exploration of pedestrian gap-acceptance behavior at selected locations," Transportation Research Record: Journal of the Transportation Research Board, no. 1982, pp. 132-140, 2006.
[16] X. Zhuang and C. Wu, "Modeling pedestrian crossing paths at unmarked roadways," IEEE Transactions on Intelligent Transportation Systems, vol. 14, no. 3, pp. 1438-1448, 2013.

[17] T. Wang, J. Wu, P. Zheng, and M. McDonald, "Study of pedestrians' gap acceptance behavior when they jaywalk outside crossing facilities," in Proceedings of the 13th International IEEE Conference on Intelligent Transportation Systems, ITSC 2010, pp. 1295-1300, IEEE, September 2010.

[18] M. Asano, T. Iryo, and M. Kuwahara, "Microscopic pedestrian simulation model combined with a tactical model for route choice behaviour," Transportation Research C: Emerging Technologies, vol. 18, no. 6, pp. 842-855, 2010.

[19] W. Zeng, P. Chen, H. Nakamura, and M. Iryo-Asano, "Application of social force model to pedestrian behavior analysis at signalized crosswalk," Transportation Research Part C: Emerging Technologies, vol. 40, pp. 143-159, 2014.

[20] N. B. Cao, Z. W. Qu, Y. H. Chen, L. Y. Zhao, X. M. Song, and Q. W. Bai, "Destination and route choice models for bidirectional pedestrian flow based on the social force model," IET Intelligent Transport Systems, pp. 1751-9578, 2017.

[21] M. Li, F. Shi, and D. Chen, "Analyze bicycle-car mixed flow by social force model for collision risk evaluation," in Proceedings of 3rd International Conference on Road Safety and Simulation, 2011. 


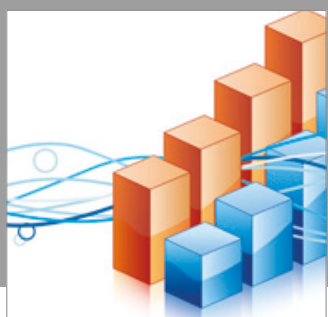

Advances in

Operations Research

vatersals

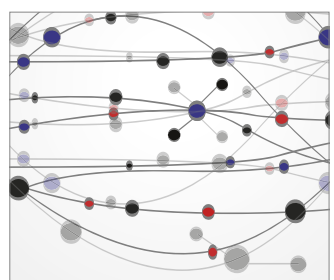

\section{The Scientific} World Journal
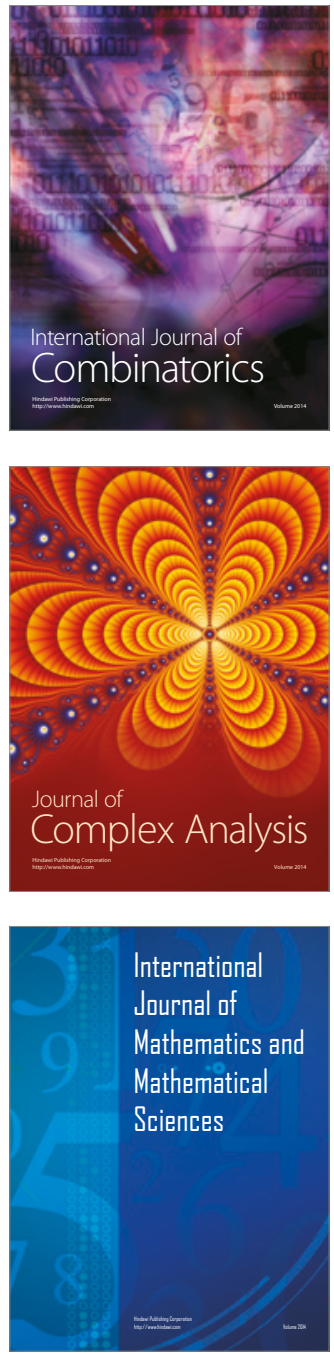
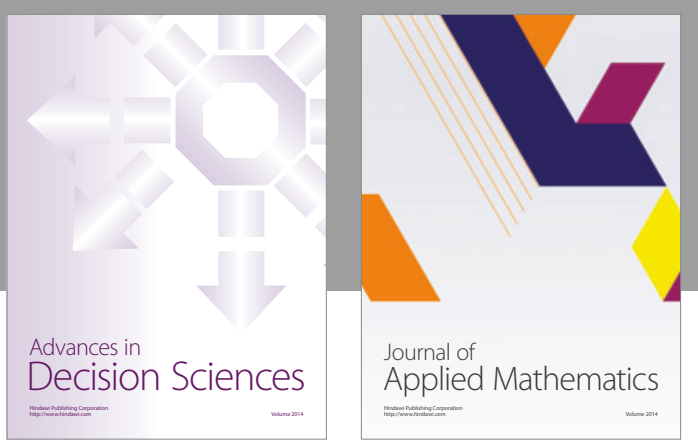

Algebra

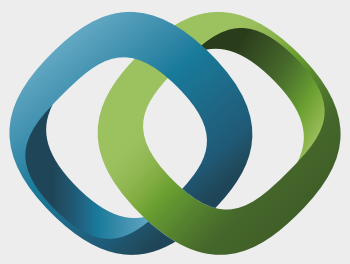

\section{Hindawi}

Submit your manuscripts at

https://www.hindawi.com
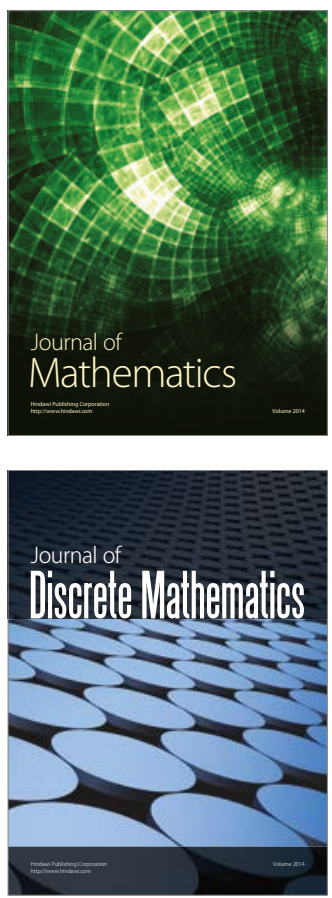

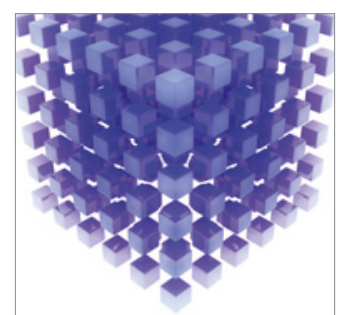

Mathematical Problems in Engineering
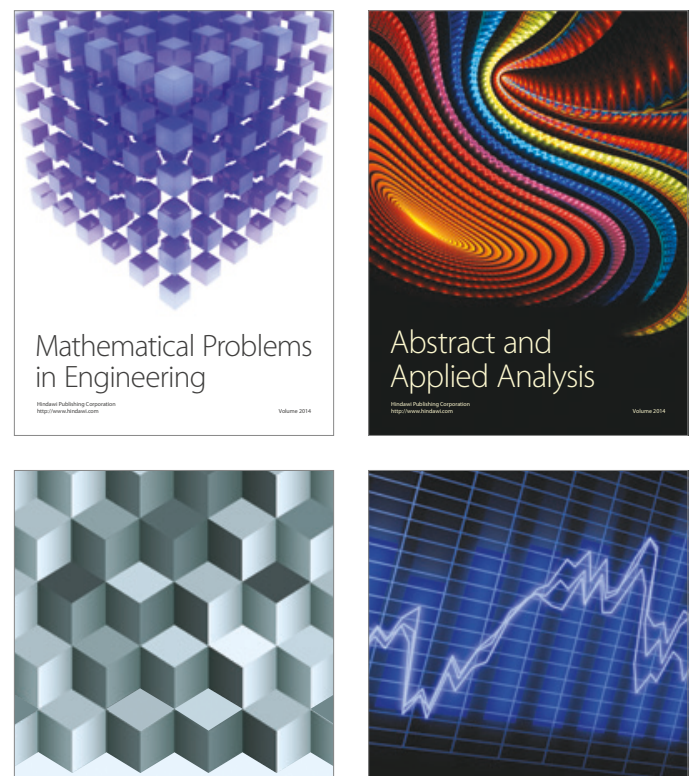

Journal of

Function Spaces

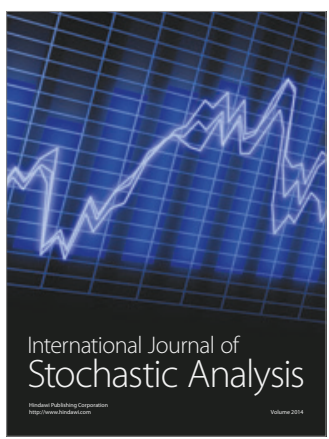

Probability and Statistics
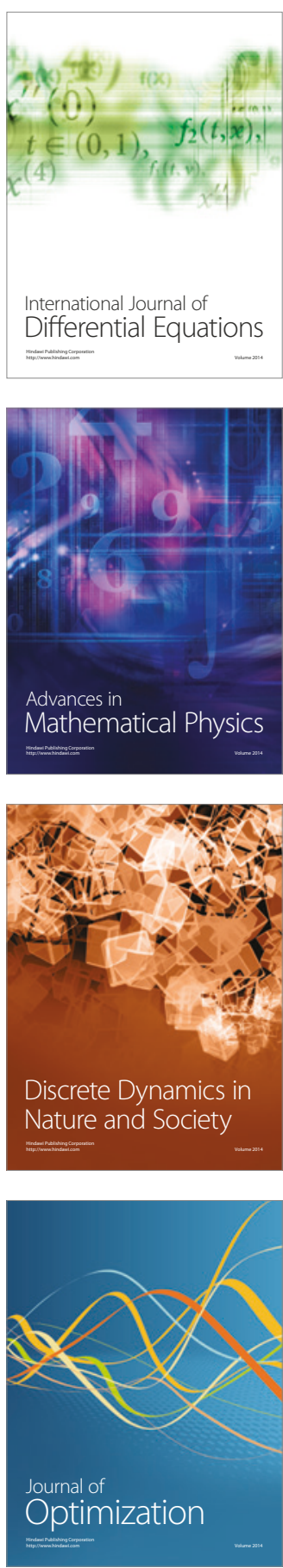